\title{
CARTOGRAFÍAS
}

\section{Antropología y diversidad sexual y de género en España. Hacia la construcción de una especialidad disciplinaria}

\author{
Anthropology and Sexual an Gender Diversity in Spain. \\ Towards the Construction of a Disciplinary Speciality
}

\author{
Jose Antonio Langarita Adiego ${ }^{1}$ \\ Universitat de Girona \\ Jordi Mas Grau ${ }^{2}$ \\ Universitat de Barcelona
}

\section{RESUMEN}

El estudio de la sexualidad desde una mirada social y cultural ha adquirido una gran relevancia en las ciencias sociales y humanas durante las últimas décadas. Este hecho se ha visto reflejado en un creciente número de etnografías y análisis antropológicos que dotan de nuevos significados a la diversidad sexual y de género más allá de las lecturas clínicas y biomédicas. En este artículo se ha efectuado una revisión bibliográfica exhaustiva con el objetivo de analizar el recorrido, las transformaciones y los ámbitos de interés de los trabajos sobre diversidad sexo-genérica en la antropología española desde 1980 hasta 2017. Veremos que, actualmente, esta especialidad disciplinaria cuenta con una amplia variedad de temáticas, ya que aparte de los estudios sobre la homosexualidad han florecido nuevas áreas de interés, tales como los estudios trans e intersex. Con este trabajo se pretende contribuir a la construcción de la historia de la antropología de la diversidad sexual y de género española desde una perspectiva situada. Este artículo demuestra que, a pesar de la inequívoca influencia internacional en el desarrollo de la especialidad en España, también existe un recorrido propio fuertemente influenciado por el contexto socio-histórico y la antropología feminista del país.

Palabras clave: Antropología de la sexualidad; Etnografía española; Diversidad sexual; Género; Sexualidad.

\footnotetext{
${ }^{1}$ Correo electrónico: josan.langarita@udg.edu. ORCID iD: http://orcid.org/0000-0002-7911-3109.

${ }^{2}$ Correo electrónico: jordimasgrau@ub.edu. ORCID iD: http://orcid.org/0000-0002-3414-9335.
} 


\section{SUMMARY}

The study of sexuality from a social and cultural standpoint has acquired great importance in the social and human sciences in recent decades. This is reflected in a growing number of ethnographies and anthropological analyses that provide a new understanding of sexual and gender diversity that goes beyond clinical and biomedical interpretations. This article is the product of an exhaustive review of the literature aimed at analysing the paths taken, the transformations effected and the areas of interest relating to work on sexual and gender diversity in Spanish anthropology from 1980 to 2017. Currently, this specialised discipline comprises a wide variety of subjects since, in addition to studies on homosexuality, there has been a rapid expansion in new areas of interest, such as trans and intersex studies. The aim of this work is to contribute to the construction of the history of anthropology of sexual and gender diversity in Spain from a situated perspective. This article demonstrates that despite the indisputable international influence on the development of this specialty in Spain, there have also been specific local developments heavily influenced by the socio-historical context and feminist anthropology in the country.

Key words: Anthropology of Sexuality; Spanish Ethnography; Sexuality; Sexual Diversity; Gender.

\section{INTRODUCCIÓN}

Que la sexualidad no es un hecho meramente natural, sino una dimensión del ser humano embebida de significaciones socio-culturales es una tesis que ha sido sostenida por numerosos trabajos provenientes de distintas disciplinas (cf. Fausto-Sterling 2000; Foucault 1976; Haraway 1991; Herdt 1997; Laqueur 1990; Nieto Piñeroba 2003; Rubin 1975 y 1984; Sedgwick 1990; Stolcke 2000; Tiefer 1995; Weeks 1985). Cada sociedad significa y regula la sexualidad en función de sus propios patrones, determinando tanto las conductas consideradas legítimas como las sancionables (Plummer 1984). Si la sexualidad es un hecho inherentemente cultural que vertebra las relaciones sociales, debería haber sido uno de los objetos clásicos de la antropología. No obstante, y a pesar de que Malinowski (1927 y 1929), Benedict (1939) y Mead (1950) habían abordado las cuestiones sexuales en tanto que manifestaciones culturales, la sexualidad estuvo prácticamente ausente de las investigaciones antropológicas hasta el último cuarto del siglo XX. Durante ese periodo de ostracismo, el estudio de la sexualidad fue propiedad casi exclusiva de las ciencias psicológicas, médicas y biológicas. Para la antropología, la sexualidad era un fenómeno, rodeado de tabúes, que pertenecía exclusivamente al ámbito individual y privado (Nieto Piñeroba 1993). Esta falta de interés —o abierto recelo— se intensificaba todavía más ante aquellas prácticas y expresiones sexo-genéricas que escapaban de la lógica androcéntrica, heterosexual y binaria. No fue hasta los años setenta que algunas investigadoras e investigadores, jugándose su reputación profesional, se interesaron por la diversidad sexual y retomaron la mirada antropológica como estrategia para conocer las formas de organización social de la sexualidad (Weston 1993).

Este artículo tiene por objetivo contribuir a la construcción de una historia de la antropología de la sexualidad y de la diversidad sexo-genérica en España. Se analiza el lugar e impacto de estos estudios dentro de la disciplina, se determina su recorrido y transformaciones en los últimos treinta y siete años, y se calibra su trascendencia para pensar la realidad social contemporánea. Veremos que, en España, las primeras 
publicaciones sobre la temática no vieron la luz hasta los años ochenta y su apogeo se produjo en el siglo XXI. En esta evolución histórica han ejercido una gran influencia los autores, corrientes y trabajos extranjeros, muy especialmente los provenientes de Francia y del mundo anglosajón. Sin embargo, la antropología de la sexualidad y de la diversidad sexo-genérica española tiene su propio recorrido, debido a factores socio-políticos de amplio alcance, como la dictadura franquista y la transición a la democracia, pero también a factores de índole académica e intelectual, como el influjo de los estudios feministas españoles. Por tanto, tanto ésta como otras historias deben siempre abordarse desde una perspectiva situada.

\section{CONSIDERACIONES METODOLÓGICAS}

En este texto se ha efectuado una revisión bibliográfica exhaustiva de las producciones etnográficas y antropológicas que, mediante monografías, compilaciones editadas y artículos en revistas científicas especializadas, han puesto su atención en la sexualidad y la diversidad sexo-genérica en el contexto español. Hemos seguido el recorrido que empieza con aquellas personas que hoy podríamos considerar pioneras en el estudio de la sexualidad, hasta quienes actualmente centran sus investigaciones en cuestiones relacionadas con la diversidad sexual y de género. Y todo ello con la finalidad de analizar, de modo diacrónico, los cambios acaecidos en este ámbito disciplinario. Este trabajo es, por tanto, una revisión crítica del progreso y actual estado de la antropología de la sexualidad en España y sigue la tradición de las publicaciones de análisis bibliográfico, como es el caso de los famosos artículos de Weston (1993) y Boellstorff (2007) sobre los estudios gays, lesbianos y trans en la antropología anglófona, o de la revisión de Frankis y Flowers (2009) sobre las investigaciones cualitativas en materia de sexo entre hombres en espacios públicos.

Para la identificación de la bibliografía especializada, se ha recurrido a la consulta de tres bases de datos españolas: Dialnet de la Universidad de la Rioja, ReCercador de la Universidad de Barcelona y la base ISOC del Consejo Superior de Investigaciones Científicas (CSIC). Con el objetivo de evitar redundancias cuando se habla de autores con una extensa producción (como sería el caso de Jose Antonio Nieto o de Oscar Guasch), se ha dado preeminencia a sus obras monográficas antes que a sus artículos y capítulos de libro, siempre y cuando estos últimos no hayan supuesto una aportación significativa a su trayectoria intelectual. En cuanto a la consideración de una publicación en tanto que perteneciente o no a la antropología, se ha priorizado la formación y/o adscripción académica del autor o autora, y la etnografía como técnica de recogida de los datos que fundamentan una obra. Con todo, y tal y como se verá a lo largo del artículo, en el estudio de la sexualidad y la diversidad sexo-genérica la antropología mantiene fuertes conexiones con otras ciencias sociales y humanas y también con el activismo LGTB+, por lo que son frecuentes las ediciones y compilaciones interdisciplinares y las que conjugan activismo y academia. En estos casos, se han seleccionado aquellas obras conjuntas en las están presentes algunas aportaciones significativas para la antropología, aunque la persona editora/compiladora no pertenezca a esta disciplina. Asimismo, y con el fin de destacar que la antropología de la sexualidad no puede entenderse sin estas conexiones e influjos mutuos, se 
ha añadido un apartado que recoge los autores y obras más relevantes procedentes de otras disciplinas y de la lucha política socio-sexual.

\section{UN EMERGENTE CAMPO DE ESTUDIO DENTRO DE UN NUEVO CONTEXTO SOCIO- POLÍTICO}

En España, el establecimiento de la dictadura franquista y las consecuencias socioeconómicas de la post-guerra afectan gravemente a las investigaciones etnográficas, dominadas hasta entonces por el folclorismo de corte romántico-naturalista. Durante los primeros años de la dictadura, los pocos trabajos etnográficos existentes proceden de distintos institutos vinculados al Consejo Superior de Investigaciones Científicas (CSIC), el órgano que crea, en 1944, la Revista de Dialectología y Tradiciones Populares (Aguirre 2015). En la década de los cincuenta, gracias al regreso del exilio de figuras como José Miguel de Barandiarán, Claudio Esteva y Julio Caro Baroja, se retoma el interés por una nueva antropología cultural (Prat i Carós 1992). Estos pioneros se centran en el estudio del patrimonio cultural español, no estando las cuestiones sexuales ni de género entre sus temas de interés.

En la España franquista y católica, la sexualidad es concebida exclusivamente como matrimonial y reproductiva. Más allá del lecho conyugal, es condenada por moralistas y científicos y perseguida por las autoridades. En un contexto socio-político en el que se reprime toda expresión sexo-genérica que no se ajusta a la norma imperante, casi no hay espacios para reflexionar sobre los usos y significados de la sexualidad y el género ${ }^{3}$. Durante esa época, autoridades franquistas como Vallejo Nágera (1937 y 1941), jefe de los Servicios Psiquiátricos Militares y miembro de la Real Academia Nacional de Medicina, legitiman la moral dominante mediante discursos pretendidamente científicos. Vallejo Nágera trata de demostrar la inferioridad biológica de diferentes grupos sociales (comunistas, mujeres y hombres poco viriles), defiende la eugenesia para purificar la raza ibérica y clama por el establecimiento de medidas para hacer frente a los instintos perversos. Para Vallejo Nágera, los regímenes democráticos son incapaces de establecer un control severo sobre las relaciones sexuales que escapan a la lógica heterosexual y reproductiva, y debilitan la moral colectiva al plegarse ante las proclamas feministas a favor del sufragio universal: "Tiene la democracia el grave inconveniente de que halaga las bajas pasiones y de que concede iguales derechos sociales al loco, al imbécil y al degenerado. El sufragio universal ha desmoralizado a las masas" (Vallejo Nagéra 1937: 129).

Otros autores como Mauricio Carlavilla (1956), Antonio Sabater Tomas (1962) o José López Ibor (1968) también contribuyen notablemente a elaborar un discurso sancionador sobre la sexualidad no reproductiva recurriendo a una retórica pseudocientífica.

${ }^{3}$ La persecución y condena de la disidencia sexo-genérica fue posible gracias a la modificación de la Ley de Vagos y Maleantes de 1953, con la que se incluyó a los homosexuales entre los sujetos considerados como antisociales. Más tarde, en 1970, se refundó en la Ley sobre Peligrosidad y Rehabilitación Social (LPRS), con la que se mantuvieron las sanciones hacia las personas homosexuales. Cabe señalar que, en 1983, se modifica la LPRS para que la homosexualidad deje de considerarse un escándalo público. No obstante, la derogación de todo el articulado relativo a homosexualidad se produce en 1989 y la derogación total de la ley en 1995. 
En el caso de López Ibor, su adhesión al movimiento nacional le permite dirigir el Centro Neurológico Militar y, posteriormente, el Servicio de Neuropsiquiatría del Hospital General de Madrid (Adam Donat y Martínez Vidal 2008). En los textos de López Ibor se detecta un cambio de conceptualización de las "aberraciones sexuales" con respecto a la obra de Vallejo Nágera que resulta fundamental, pues presenta la homosexualidad no ya como un acto delictivo, sino más bien como una enfermedad que ha de ser tratada en instituciones terapéuticas con el fin de proteger a la sociedad de sus efectos devastadores. Este cambio conceptual permite el desarrollo de las terapias curativas de la homosexualidad, mediante las cuales se obliga al paciente a ingerir sustancias que provocan vómitos o a sufrir descargas eléctricas mientras es expuesto a estímulos homosexuales (Arnalte 2003: 100).

Con la muerte de Franco se inicia en España la transición a la democracia. Este periodo se caracteriza por la instauración de una monarquía parlamentaria, el establecimiento de las instituciones democráticas y el ingreso de España en organismos internacionales como la OTAN (en 1982) y la Comunidad Económica Europea (en 1986). Además, la derogación de la mayoría de las leyes represivas del franquismo debilita la persecución jurídica de las minorías sexo-genéricas, las cuales empiezan a adquirir cierta visibilidad y a organizarse formalmente para reclamar derechos ${ }^{4}$. En un corto espacio de tiempo se producen diferentes hechos de relevancia para las minorías sexogenéricas: en 1975 se funda el Front d'Alliberament Gai de Catalunya, primera organización visible que defiende la liberación sexual (es legalizado en 1980), tiene lugar la primera manifestación por la liberación homosexual en Barcelona (1977) y aparece la primera asociación trans (1978). En definitiva, una multitud de discursos, cuerpos y actos heterodoxos empiezan a desafiar la moral católica hegemónica, omnipresente hasta ese momento.

Por otra parte, el proceso de institucionalización de la antropología en España se inicia unos años antes del fin del franquismo. A inicios de los años sesenta, José Alcina Franch crea el seminario de antropología americana y un curso introductorio de antropología en la Universidad de Sevilla. Poco tiempo más tarde, en 1966, Claudi Esteva Fabregat funda la Escuela de Estudios Antropológicos en la sede del Museo Etnográfico de Madrid y contribuye decisivamente a la creación del primer departamento de antropología cultural en la Universitat de Barcelona, en 1968. Es partir de entonces cuando las universidades españolas empiezan a dotarse de titularidades y cátedras de antropología (Aguirre 2015; Capel 2009).

En este clima de cambios políticos, sociales e institucionales aparecen figuras como las del antropólogo Alberto Cardín, uno de los primeros intelectuales homosexuales de la España postfranquista. A pesar de que Cardín se muestra especialmente fascinado por el estudio de las religiones, también introduce los análisis sobre la sexualidad en la emergente antropología española, muy especialmente con Guerreros, Chamanes $y$ Travestis (1984). En esta obra de referencia analiza diferentes formas de expresión sexo-genérica características de otras sociedades, entre las que destacan algunas figuras institucionalizadas de variancia de género, como el berdache norteamericano. Asi-

\footnotetext{
${ }^{4}$ Cabe señalar que los artículos relativos a la homosexualidad de la Ley de Peligrosidad y Rehabilitación Social no fueron derogados hasta enero de 1979, y la derogación total se llevó a cabo en 1995.
} 
mismo, el autor se muestra especialmente crítico con el precepto militante de la época, que pretende justificar la lucha del movimiento gay mediante referencias a la experiencia homosexual en otras culturas. En opinión de Cardín, esta estrategia genera un grave problema metodológico, ya que lo único que pretende es confirmar "mediante la acumulación de ejemplos verosímiles, una realidad aceptada de partida" (Cardín 1984: 19). Es decir, desde el punto de vista antropológico no es posible equiparar un fenómeno occidental y contemporáneo, como la homosexualidad, con prácticas y expresiones sexo-genéricas de otras realidades culturales e históricas. Más adelante, promueve la crítica social a la perspectiva médica y periodística sobre el VIH (Cardín y Fluvià 1985; Cardín 1991) y publica diferentes artículos de divulgación y relatos en los que aborda sin tapujos la cuestión homosexual. Cardín también traduce al español y prologa el Diario de campo en Melanesia, una de las obras más controvertidas de Malinowski, puesto que en ella el maestro de la antropología hace referencia a algunos sueños homoeróticos que le acechan durante su trabajo de campo:

Hoy lunes, 20 de septiembre de 1914 tuve un extraño sueño homosexual con mi propio doble como pareja. Extrañas sensaciones homoeróticas; la impresión de ir a besar una boca como la mía, un cuello torneado como el mío, una frente idéntica a la mía (Malinowski 1989: 41).

La muerte de Cardín en 1992, víctima del sida, acaba prematuramente con una figura pionera y prometedora para la antropología, la literatura y la cultura en general, ya que también se le conoce por sus trabajos poéticos y narrativos, así como por su aparición frecuente en los medios de comunicación. En palabras de Manuel Delgado (1992), Cardín "fue sobre todo un transgresor, dedicado a lanzar con sus textos golpes de mano contra la cultura española oficial».

En los años ochenta aparece en escena José Antonio Nieto, formado en la New School for Social Research de Nueva York, y a quien se le podría atribuir, junto con Alberto Cardín, la paternidad de la antropología de la sexualidad en España. Nieto acerca el estudio de la sexualidad a unos académicos todavía recelosos de incorporar este campo a las ciencias sociales. De entre su obra en español destaca, en primer lugar, Cultura y sociedad en las prácticas sexuales (1989), elaborada con el fin de servir de manual para el Máster en Sexualidad Humana, organizado por la Universidad Nacional de Educación a Distancia (UNED). Con este máster, que se prolonga hasta el año 2000, una universidad española imparte por vez primera unos estudios oficiales sobre la sexualidad desde una perspectiva sociocultural, y no solo biomédica (Calle 2011). Redactado con un estilo pedagógico, en este manual Nieto analiza la dimensión antropológica de la sexualidad y presenta algunas de las cuestiones relevantes para la especialidad (como el coito, la prostitución, las relaciones homosexuales o la inversión de los géneros), apoyándose en numerosos casos etnográficos. Poco más tarde ejerce de compilador de otro volumen destinado al máster de la UNED, La sexualidad en la sociedad contemporánea. Lecturas antropológicas (1991), y publica Sexualidad y deseo. Crítica antropológica de la cultura (1993). Progresivamente, Nieto va obteniendo el reconocimiento de sus colegas de profesión, hecho que contribuye a que la sexualidad sea aceptada como una especialidad dentro de la antropología. Asimismo, con Transexualidad, transgenerismo y cultura (1998) Nieto se convierte en el primer antropólogo que compila y traduce al español algunos de los principales 
textos que abordan el transgenerismo desde una óptica social. En sus escritos posteriores, entre los que sobresalen Antropología de la sexualidad y diversidad cultural (2003), Transexualidad, intersexualidad y dualidad de género (2008) y Sociodiversidad y sexualidad (2011), Nieto da continuidad a su itinerario intelectual analizando las corrientes teóricas sobre la sexualidad predominantes en ciencias sociales e interesándose por los fenómenos de variancia de género — como el hijra indio o el mabu tahitiano- en contextos no occidentales. El abordaje de estas figuras transgenéricas sirve de paso a Nieto para efectuar una de sus mayores contribuciones a la especialidad, pues introduce en España la crítica de nuestro sistema de sexo/género dicotómico y excluyente a partir del cuestionamiento del paradigma biomédico que patologiza y medicaliza las expresiones trans e intersex.

\section{LA CONSOLIDACIÓN DE UNA ANTROPOLOGÍA DE LA SEXUALIDAD EN ESPAÑA}

A lo largo de los años ochenta y principios de los noventa se vive lo que podríamos llamar la transición sexual española. La desaparición de la censura, al menos formalmente, y los deseos de aperturismo sexual explican fenómenos como los del destape, a saber, la exposición del cuerpo desnudo o semidesnudo - muy especialmente el femenino- en el cine (con comedias pícaras), en las revistas de contenido erótico y también en las playas españolas ${ }^{5}$. La sexualidad y las relaciones sexo-amorosas empiezan a ocupar el espacio televisivo con programas de gran audiencia ${ }^{6}$. El tipo de sexualidad presente en los medios de comunicación es eminentemente heterosexual y androcéntrico, por lo que las expresiones sexo-genéricas disidentes tienden a ser visibilizadas con fines jocosos y/o condenatorios. Ello explica la sobreexposición a la que son sometidas algunas figuras transgénero, convertidas en iconos del exceso y la extravagancia por programas nocturnos marcadamente sensacionalistas (Guasch Andreu y Mas Grau 2014)7. En el mundo del cine, algunos directores españoles de la época explicitan en sus películas las cuestiones sexuales. Entre ellos destacan Pedro Almodóvar o Ventura Pons, que en sus filmes acostumbran a dar protagonismo a personajes homosexuales y transgénero. En el ámbito literario florecen autores que ofrecen otras miradas del género y la sexualidad, como es el caso de Juan Goytisolo (1985), Carmen Martín Gaite (1992), Eduardo Mendicutti (1982), Luis Antonio de Villena (1982 y 1989), Esther Tusquets (1978) o Manuel Vázquez Montalbán (1987), entre otros.

Asimismo, la diversidad sexual se hace cada vez más visible en las grandes ciudades españolas. En ellas proliferan los colectivos de defensa de los derechos de las personas LGTB y la celebración del 28 de junio se convierte en un acto habitual de los grandes centros urbanos del país 8 . Poco a poco aparecen zonas de ocio y de socialización dirigidas a la población gay, como la zona del Gaixample en Barcelona o

\footnotetext{
${ }^{5}$ Destacan revistas como Interviú, Lib o Party.

${ }^{6}$ Como, por ejemplo, iAy, qué calor!, Tutti Frutti o Hablemos de sexo.

7 Es el caso de personajes trans como la Veneno o Carmen de Mairena y de los programas nocturnos Esta noche cruzamos el Mississippi y Crónicas Marcianas.

${ }^{8}$ El 28 de junio se conmemoran las revueltas de Stonewall (Nueva York) de 1969.
} 
el barrio de Chueca en Madrid. Pero la pandemia del sida es lo que sitúa la cuestión homosexual en la arena pública, por lo que la visibilización de la población gay está rodeada de dudas, sospechas y críticas.

La sociedad española de la época facilita que la diversidad sexo-genérica se convierta en una área de interés para unas ciencias sociales con tendencia a investigar la desviación. Además, en países como Francia, y especialmente en el Reino Unido y Estados Unidos, los estudios LGTB van consolidándose en el entorno académico (cf. Weston 1993), lo que sirve de estímulo para que las académicas y académicos españoles se acerquen a este campo de estudio. Pero si bien Cardín y Nieto ya se habían aproximado a las sexualidades y expresiones de género no hegemónicas desde la antropología de los años ochenta, todavía no existían en ese momento etnografías que abordasen el fenómeno desde una perspectiva situada.

Esta realidad cambia en 1991, cuando Óscar Guasch Andreu publica La sociedad rosa, la primera etnografía española dedicada exclusivamente a la cuestión gay. En esta obra, la población homosexual es concebida como una subcultura con prácticas y significados propios y, por tanto, susceptible de ser analizada con métodos cualitativos. Guasch Andreu estudia las formas de clasificación social de la homosexualidad, así como las interacciones producidas en diferentes espacios de socialización gay de finales de los ochenta y principios de los noventa. Asimismo, identifica dos fases fundamentales en la construcción de la identidad gay española: el periodo pregay (que abarca los años del franquismo y los primeros tiempos de la transición a la democracia), en el que la homosexualidad tan solo puede pensarse tomando como referente a la heterosexualidad, al tiempo que el homosexual es identificado socialmente con lo femenino; y el periodo gay (iniciado en los años 80), en el que los homosexuales convierten su diferencia en orgullo e inician un proceso de integración social, si bien se ven obligados a repensar sus prácticas a causa del miedo generado por el sida.

Pocos años después se publica el libro de Begonya Enguix (1995) Poder y deseo: la bomosexualidad masculina en Valencia, donde la autora explora las percepciones y categorías vinculadas a la homosexualidad masculina en dicha ciudad. Posteriormente, Enguix ha seguido divulgando sus estudios sobre el mundo gay español en varios artículos académicos, centrando su atención en cuestiones relacionadas con las producciones del cuerpo masculino y sus espacios de representación (2010, 2012 y 2017). Por su parte, Joan Vendrell i Ferré publica en 1994 un artículo en el que desarrolla unos principios etnográficos generales para la investigación cualitativa de la sexualidad (Vendrell i Ferré 1994), y más adelante reflexiona sobre la construcción del cuerpo sexuado y de las identidades sexuales y de género (Vendrell i Ferré 1999). Los trabajos de Guasch, Enguix y Vendrell provienen del Departamento de Antropología de la Universidad Rovira i Virgili de Tarragona, convertido en todo un referente nacional en el estudio de la sexualidad durante los años noventa.

Aparte de los trabajos de Guasch Andreu, Enguix y Vendrell, no abundan las investigaciones sobre diversidad sexual y de género en la España de los noventa. En el caso de la sociología, la situación no es muy distinta. Como señala Guasch Andreu (1993), los pocos trabajos existentes analizan las sexualidades periféricas, como la prostitución (Negre 1988; Osborne 1991) o la homosexualidad (Bonal, Climent y Costa 1985; Rodríguez 1985). Mención especial merece el artículo de Iglesias de Ussel (1983), publicado en la revista REIS con un título revelador: "La sociología de la sexua- 
lidad en España. Notas introductorias». En este trabajo el autor efectúa una breve historia - en especial del siglo XX- del tratamiento dado a la sexualidad en España, describe el estado de las investigaciones de la época sobre esta temática y realiza un alegato en favor del abordaje de la sexualidad desde las ciencias sociales.

Con todo, durante esa década se consolida una antropología feminista que va elaborando discursos propios gracias al compromiso de antropólogas como Teresa del Valle (1993 y 2000; Valle y Sanz 1991), Verena Stolcke (1992 y 2000), Dolores Juliano (1992 y 1998) o Dolors Comas d'Argemir (1990 y 1995). En la España de los noventa, el mundo académico todavía desconfía de la sexualidad como objeto de estudio legítimo para las ciencias sociales, e ignora el impacto del feminismo sobre el resto de ámbitos de investigación. Por todo ello, la importancia de estas investigadoras no solo se debe a que forman e inspiran a toda una generación de antropólogas feministas, sino también a que contribuyen a la construcción de una teoría social de la sexualidad, a la inclusión de las cuestiones relacionadas con la diversidad sexo-genérica en el panorama académico español y al cuestionamiento de una academia androcéntrica que infravalora tanto el papel de las intelectuales como la mirada femenina - antipatriarcal- en la investigación social.

Al influjo del feminismo debemos sumarle otros dos factores que son determinantes para estimular el interés por la sexualidad y la diversidad sexual y de género en la antropología española. El primero de ellos es la traducción al español de algunos textos clásicos internacionales de la especialidad, como los trabajos de Gayle Rubin (1986 y 1989), Gilbert Herdt (1992) o Kath Weston (2003). El segundo factor es la irrupción, en los noventa, del pensamiento queer, que aporta nuevas perspectivas y problematizaciones a los estudios sobre la sexualidad y el género. Los trabajos de Butler (1990 y 1993), Sedgwick (1990), Haraway (1991) o De Lauretis (1994), muy influenciados por el pensamiento francés de Foucault, Derrida y Wittig, son también una referencia para los antropólogos y antropólogas españoles que deciden investigar sobre estas cuestiones desde las ciencias sociales y humanas. A pesar de que no todas las investigaciones desarrolladas en el siglo XXI son abiertamente queer, todas ellas, de una manera $\mathrm{u}$ otra, tienen que lidiar con los temas que esta corriente de pensamiento ha convertido en prácticamente ineludibles: la problematización de las categorías sexogenéricas, la naturaleza y construcción del género (con la teoría de la performatividad butleriana a la cabeza) o la producción de hegemonías y normatividades sexo-genéricas.

Es a principios del siglo XXI cuando se multiplican los trabajos sobre diversidad sexual y de género en la antropología española. Olga Viñuales (2000) publica la primera etnografía sobre el lesbianismo en España, en la que estudia el proceso de adscripción a la categoría "lesbiana" y el modo en que estas mujeres negocian su identidad sexual en diferentes escenarios y grupos sociales, como el trabajo, la familia o los círculos de amistad. Posteriormente, Viñuales se interesa por las prácticas y relaciones BDSMํ (Sáez Jiménez y Viñuales 2007) y, junto con Guasch Andreu, edita un volumen titulado Sexualidades: diversidad y control social (2003). Dicho volumen está conformado por una amplia variedad de contribuciones con temáticas tan diversas como la intersexualidad, la pornografía, la prostitución o los dispositivos y técnicas heterose-

\footnotetext{
${ }^{9}$ BDSM es el acrónimo empleado para referirse a las prácticas de Bondage, Disciplina, Dominación, Sumisión, Sadismo y Masoquismo.
} 
xualizantes, lo que ayuda a abrir la mirada de la diversidad sexual más allá de las relaciones homosexuales. Guasch Andreu realiza otros trabajos, entre los que destacan un ensayo crítico sobre la construcción social de la heterosexualidad, en donde problematiza las lógicas sexuales normativas (Guasch Andreu 2000), y un libro en el que identifica cuatro formas ideales de masculinidad con el objetivo de mostrar no solo jerarquías y subalternidades, sino también la inestabilidad y carencias de la masculinidad hegemónica (Guasch Andreu 2006). Más recientemente, Guasch Andreu y Lizardo (2017) publican un trabajo en el que analizan la prostitución masculina en diferentes espacios sociales.

También en este periodo, Fernando Villaamil (2004) publica un libro en el que retoma la cuestión de la identidad gay para actualizar el debate en función de las nuevas condiciones sociales del país y del impacto del VIH. Tras su primer libro, Villaamil realiza sustanciales contribuciones a la antropología de la sexualidad, tanto en solitario como con María Isabel Jociles y Fernando Lores, a partir del estudio de las enfermedades de transmisión sexual de hombres que tienen sexo con hombres (Villaamil Pérez, Jociles Rubio y Lores Masip 2005; Villaamil Pérez y Jociles Rubio 2008 y 2011; Villaamil Pérez 2013 y 2016).

El VIH ha tenido un gran impacto sobre la población homosexual. La pandemia no solo ha acabado trágicamente con la vida de muchas personas, sino que también ha alterado significativamente las prácticas sexuales, las estrategias relacionales, las representaciones y los discursos sobre la sexualidad, muy especialmente entre la población homosexual. No obstante, en España, salvo algunas excepciones mencionadas, son escasos los trabajos que han abordado este fenómeno desde una perspectiva sociocultural, concediendo así a la biomedicina el espacio privilegiado para la generación de discursos expertos sobre la enfermedad.

José María Valcuende del Río es otro de los autores de referencia que empieza a destacar en los primeros años del siglo XXI. Aunque en muchas ocasiones se le ha relacionado con la antropología ecológica, también sobresalen sus aportaciones a la antropología de la sexualidad. Junto con Juan Blanco edita uno de los primeros libros en España dedicado al estudio de las masculinidades, en el que participan autores y autoras provenientes de distintas ciencias sociales y humanas (Blanco López y Valcuende del Río 2003). En este volumen se encuentra un destacado artículo de Valcuende, elaborado junto a Assumpta Sabuco, en el que analizan la representación de la masculinidad homosexual contemporánea y efectúan una crítica radical de las formas de masculinidad gay (Valcuende del Río y Sabuco i Cantó 2003). Valcuende ha sido también el promotor del Laboratorio Iberoamericano de estudios sociohistóricos de la sexualidad (la red LIESS), formado por más de setenta investigadores e investigadoras, y ha coorganizado las tres primeras ediciones del Congreso internacional sobre diversidad sexual en Iberoamérica. Cabe señalar que, aunque la red LIESS no está formada exclusivamente por antropólogas y antropólogos, éstos han encontrado en ella un espacio de discusión que les ha permitido establecer lazos entre Latinoamérica y España para pensar la diversidad sexual y de género desde perspectivas hispanohablantes. Otras aportaciones destacables son las de Carmen Gregorio (1998 y 2014) y Mari Luz Esteban (2004 y 2011), quienes a pesar de no centrar su trabajo exclusivamente en la sexualidad, mantienen una amplia mirada que les permite acercarse a la diversidad sexual y de género desde los estudios corporales y la perspectiva feminista. 
Por otra parte, la aprobación del matrimonio y la legalización de la adopción entre personas del mismo sexo, en el año 2005, ha generado abundantes debates y propuestas. Se han abierto nuevos horizontes de investigación $-\mathrm{y}$ entrecruzamientos entre las antropologías del parentesco, del género y de la sexualidad- que visibilizan las múltiples formas de entender la familia, la pareja y las relaciones paterno y maternofiliales en España (cf. Donoso López 2006 y 2012; Falguera Ríos 2013; Imaz 2010; Lores Masip y Ayala Rubio 2015; Pichardo Galán, Stéfano Barbero y Martín-Chiappe 2015; Pichardo Galán 2009; Roigé 2006; Valcuende del Río 2006).

En estos últimos quince años se han perfilado y asentado los debates, al tiempo que han surgido nuevas áreas de interés a medida que se ha ido reconociendo el amplísimo paraguas de la diversidad sexo-genérica. En este sentido, hay que destacar el florecimiento de los denominados estudios trans (conocidos en inglés como transgender studies), cuyos orígenes se remontan a los mundos académico y activista norteamericano de los años noventa (cf. Galofré y Missé 2015; Stryker y Aizura 2013; Stryker y Whittle 2006). La primera etnografía publicada en España sobre el fenómeno trans es la de Norma Mejía (2006), quien desde una perspectiva autoetnográfica, que ella misma califica de "etnografía extrema", efectúa un recorrido socio-histórico por el mundo de la prostitución trans barcelonesa y reflexiona sobre su propio proceso de transición. Dentro de este campo emergente, Vendrell i Ferré (2009) evalúa las consecuencias para el movimiento trans de haber reclamado a los poderes públicos que se encarguen de la gestión médico-legal de la transexualidad. Por su parte, Julieta Vartabedian (2012, 2013 y 2014) analiza la construcción de las identidades, y los tránsitos corporales y espaciales, de las travestis brasileñas que migran a Río de Janeiro y a Barcelona. Jordi Mas Grau (2014, 2015 y 2017) estudia los discursos, representaciones y prácticas generados socialmente a lo largo del proceso de (re)construcción identitaria y corporal de las personas trans, así como la gestión biomédica del transgenerismo llevada a cabo en la Unidad de Identidad de Género del Hospital Clínico de Barcelona. El cuestionamiento de la dicotomía sexo-genérica y la crítica al paradigma biomédico se ha sofisticado todavía más con los análisis de la intersexualidad de Núria Gregori (2006 y 2013) y los abordajes de las performances drag-king y las prácticas cross-dressing de Alba Barbé i Serra (2013 y 2015).

Más allá de la creciente preocupación por los fenómenos trans e intersex y otras cuestiones emergentes, algunas investigaciones mantienen el interés por lo que hoy ya son temas clásicos de la antropología de la sexualidad, como es el caso de las homosexualidades masculina y femenina. Recientes trabajos profundizan en las relaciones entre hombres para repensar las lógicas de la comunidad gay o, al menos, problematizar sus ejes vectores y formas de representación. En este sentido, aparecen en escena trabajos como el de Fernando Lores Masip (2012), quien a partir del análisis de los discursos sobre las enfermedades de transmisión sexual entre hombres, somete a discusión la forma en que se construyen las economías sexuales. Rafael Cáceres Feria y José María Valcuende del Río (2014), por su parte, estudian los procesos de hibridación entre lo local y lo global en la construcción de la homosexualidad masculina andaluza. David Berná (2016) introduce la perspectiva interseccional para analizar los procesos de construcción identitaria entre las personas gitanas LGTB. Asimismo, hay que destacar dos trabajos cuyo objeto de estudio resulta novedoso en España: la aproximación antropológica a la zoofilia de Rafael Cáceres (2013) y la primera et- 
nografía sobre sexo anónimo entre hombres, una práctica más conocida por el anglicismo cruising, de Jose Antonio Langarita Adiego (2015). En lo que respecta a las revisiones de los discursos y representaciones de las relaciones entre mujeres, cabe destacar los libros editados por Platero (2008) o Suárez Briones (2013 y 2014). Si bien ni los editores ni todos los autores de estos dos volúmenes son antropólogos, en ellos podemos encontrar contribuciones significativas para la disciplina.

ANTROPOLOGÍA, CIENCIAS SOCIALES Y HUMANAS Y ACTIVISMO LGTB+: INFLUJOS MUTUOS PARA EL ENTENDIMIENTO Y LEGITIMACIÓN DE LA DIVERSIDAD SEXO-GENÉRICA

La construcción de discursos sobre la sexualidad y la diversidad sexo-genérica en la antropología española no se ha valido únicamente de trabajos etnográficos propios de la disciplina. Este logro ha sido también posible gracias al diálogo constante y enriquecedor con otras ciencias sociales y humanas (como la filosofía, los estudios culturales, la historia, la sociología, la psicología social o la geografía), algunas de las cuales cuentan con una tradición más larga y consolidada en el estudio de estas cuestiones. Tampoco se puede olvidar que las teorías y metodologías de la antropología de la sexualidad se han nutrido del activismo LGTB+. Muy a menudo, la frontera que separa las esferas política y académica es difícil de establecer, puesto que bastantes investigadoras e investigadores se definen tanto por su actividad académica como por su lucha político-sexual.

En el caso de las ciencias sociales y humanas, sobresalen autoras como Elvira Burgos Díaz (2013 y 2014) o Paul B. Preciado (2002 y 2008), quienes exploran nuevas experiencias corporales y subjetivas desde la filosofía feminista y queer, al tiempo que problematizan el orden sexo-genérico hegemónico. En el ámbito filosófico también destaca el papel de Paco Vidarte (2007), quien a través de su particular manifiesto propone nuevas maneras de pensar la sexualidad y la política sexual, y las aportaciones de Ricardo Llamas (1995 y 1998), las cuales constituyen una de las primeras aproximaciones a la teoría queer en España y una reflexión sobre el VIH desde una perspectiva social. Desde una perspectiva próxima a la sociología histórica y filosófica, Javier Ugarte Pérez (2008 y 2014) analiza la construcción de la identidad homosexual. En cuanto a los estudios culturales, destaca la obra de Rafael M. Mérida Jiménez, el cual ha publicado un trabajo sobre la sexualidad femenina en el medievo desde una mirada renovada (2008), ha estudiado la historia de la gestión institucional de lo trans (2009a) y ha abordado la figura del travestí en España durante el Tardofranquismo y la Transición (2016). Mérida Jiménez también ha editado un par de libros con traducciones de textos anglófonos que aproximan los discursos, luchas y propuestas queer al mundo hispano (2002 y 2009b), y ha colaborado en la producción de un pensamiento español propio con varias compilaciones de estudios LGTB (Acebrón Ruiz y Mérida Jiménez 2006; Mérida Jiménez y Peralta 2015; Mérida Jiménez 2013). En el campo de los estudios culturales también destacan autoras como Meri Torras (2007 y 2011).

Desde la sociología, trabajos como los de Gerard Coll-Planas (2010 y 2012), Gracia Trujillo (2009), Kerman Calvo (2003 y 2010), Patricia Soley-Beltran (2009) o Jokin Azpiazu (2017) se han convertido en referentes para muchos antropólogos y antropólogas interesados en la diversidad sexo-genérica. Y en el ámbito de la historia, cada vez 
abundan más los estudios que trazan las diferentes genealogías de la diversidad sexual y de género en España (cf. Arnalte 2003; Cleminson y Vazquez García 2007; Huard 2014; Mira 2004; Vázquez García y Moreno Mengíbar 1997). En geografía humana destacan los trabajos de María Rodó de Zárate (2013 y 2015), en los que analiza los usos de los espacios públicos desde la perspectiva del género y la sexualidad, prestando una atención especial a las lesbianas. Por tanto, debemos reconocer que, si bien la antropología ha aportado una particular y estimulante mirada de las sexualidades y expresiones de género, dicha mirada ha podido conformarse gracias a los vínculos generados con otras áreas de conocimiento.

Asimismo, la inspiración y el trabajo de los antropólogos y antropólogas también deben mucho al activismo LGTB+, del que reciben constantes aportaciones y críticas constructivas. De las sinergias y entrecruzamientos entre la academia y el activismo han surgido publicaciones significativas, tales como el volumen El eje del mal es heterosexual (Romero Bachiller, García Dauder y Bargueiras Martínez 2005); Teoría Queer: políticas bolleras, maricas, trans, mestizas (Córdoba García, Sáez y Vidarte 2005); el provocador libro Por el culo. Políticas anales (Sáez y Carrascosa 2011); la compilación elaborada por R. Lucas Platero sobre interseccionalidad y disidencias sexo-genéricas (2012) o el volumen sobre transfeminismos editado por Miriam Solá y Elena Urko (2013). Todos estos trabajos combinan una autoría que oscila entre lo académico y lo político, desdibujando así la clásica relación de poder que se establece entre el pensamiento y la acción. Además, demuestran que las personas LGTB+, tras haber sido durante largo tiempo objeto predilecto de estudio de disciplinas como la psiquiatría, la psicología o la medicina, son hoy sujetos activos que producen un conocimiento fundamental para problematizar la lógica del sistema de sexo-género y reivindicar experiencias y cuerpos alternativos.

\section{NUEVOS HORIZONTES Y RETOS PARA LA ANTROPOLOGÍA DE LA DIVERSIDAD SEXUAL Y DE GÉNERO EN ESPAÑA}

Esta revisión de los estudios etnográficos sobre la sexualidad y la diversidad sexogenérica en España muestra el creciente interés que suscita esta temática entre las nuevas generaciones de antropólogas y antropólogos. Las reflexiones, métodos y debates surgidos de este campo de estudio suponen una gran contribución a la teoría general de la disciplina y, por extensión, a la interpretación de la realidad social. Evidentemente, sería pretencioso, aventurado y erróneo pensar que ha sido la antropología, como disciplina, el motor de las transformaciones socio-sexuales producidas en España durante las últimas décadas. Hay que atender a un sinfín de fenómenos y complejas interacciones, producidos en múltiples esferas sociales, para entender la resignificación que han experimentado la sexualidad y las relaciones de género en los últimos treinta y siete años.

Sin embargo, estos trabajos sí que han acompañado al conjunto de cambios sociales que ha experimentado España. Las antropólogas y antropólogos han abordado estos cambios para llevar a cabo análisis que permiten una mayor y mejor comprensión de la dimensión sociocultural de todo lo acontecido, problematizando las normas, patrones comportamentales y relaciones de poder mediatizados por la sexualidad y el gé- 
nero. A modo de ejemplo, la perspectiva antropológica ha contribuído al entendimiento de fenómenos como la diversificación de los modelos de familia o la visibilización de las relaciones no heterosexuales y de las identidades sexo-genéricas heterodoxas, y ha reivindicado la importancia del análisis socio-cultural de fénomenos antes exclusivamente abordados por la perspectiva biomédica, como la transexualidad, la intersexualidad o las enfermedades de transmisión sexual. En definitiva, la mirada antropológica ha ayudado a comprender el carácter social y cultural de la sexualidad y del género a través del análisis riguroso y pormenorizado que ofrece el método etnográfico.

Además, gracias al estudio de otros contextos culturales, las antropólogas y antropólogos españoles han acercado a nuestro entorno otras realidades que permiten relativizar los paradigmas dominantes fundamentados en las relaciones heterosexuales, cisgenéricas, monógamas y basados en la premisa del amor. Por su parte, las etnografías en territorio español han permitido visibilizar algunas realidades olvidadas, ignoradas o repudiadas y ofrecido miradas alternativas al relato hegemónico producido desde el mundo académico anglosajón. Asimismo, se han trasladado frecuentemente los debates surgidos de las investigaciones a los movimientos sociales y la sociedad civil. Este hecho ha permitido ampliar la visibilidad social de la disciplina, aportando herramientas para la interpretación de los fenómenos sociales a personas no iniciadas en los estudios antropológicos. No obstante, todavía queda un largo recorrido para consolidar las perspectivas antropológicas entre la población en general y entre la comunidad científica en particular, ya que los discursos biomédicos y psicológicos continúan presentándose como las interpretaciones fundamentales de la sexualidad y el género, por lo que ocupan frecuentemente un espacio preeminente en los medios de comunicación y de producción científica.

De un modo similar, el auge de los estudios sobre la sexualidad y la diversidad sexo-genérica no se ha visto reflejado en el conjunto de la antropología. Todavía hoy bastantes trabajos etnográficos no tienen presente la importancia de la dimensión sexogenérica en la configuración de las relaciones de poder, las jerarquías y las desigualdades sociales, aunque dicha dimensión resulta fundamental a la hora de determinar el acceso a los recursos, y el reconocimiento y la representación de las personas y los grupos sociales. Es más, a pesar de los avances, la antropología como disciplina todavía tiene pendiente revisar la forma con la que la mirada heterosexual y masculina impregna la comprensión de los fenómenos sociales y culturales.

Una prueba de la insuficiente presencia de las reflexiones en materia de diversidad sexo-genérica en la antropología española es la constitución de los planes de estudio de grado en las universidades españolas. Las asignaturas sobre diversidad sexual y de género, cuando están presentes, son absolutamente periféricas, y su impartición no se debe al reconocimiento de una especificidad o relevancia temática, sino más bien al empeño personal de algunos y algunas docentes. Asimismo, la carencia de revistas especializadas en lengua española es otro de los hándicaps para la expansión de este campo emergente, ya que en muchas ocasiones se trasladan las producciones a un contexto anglófono, con lo que se dificulta la divulgación de los trabajos dentro de los marcos de referencia locales.

Si nos fijamos en los programas de los congresos de antropología de los últimos años, en tanto que otro de los grandes espacios de producción y debate académico, se puede evaluar el estado y progreso de los estudios sobre la sexualidad dentro de la disci- 
plina. Por un lado, en algunos congresos se detecta una creciente visibilidad de comunicaciones y simposios que abordan temáticas claramente identificables con esta especialidad, como es el caso del primer congreso de la Asociación de Antropólogos Iberoamericanos en Red (AIBR), celebrado en Madrid en 2015. Pese a ello, la presencia de la diversidad sexo-genérica en los congresos todavía no está tan consolidada como otras temáticas antropológicas, tales como la antropología urbana, religiosa o del patrimonio. A modo de ejemplo, en el último congreso de la Federación de Asociaciones de Antropología del Estado Español (FAAEE), celebrado en Valencia en 2017, dicha presencia ha sido meramente testimonial. Además, las comunicaciones sobre diversidad sexo-genérica se incorporan frecuentemente en paneles de temáticas más amplias relacionados con el género y el feminismo. Este hecho demuestra, por un lado, las dificultades para encontrar un espacio propio y, por el otro, los intensos vínculos existentes entre la antropología feminista y los estudios sobre la diversidad sexual y de género.

Por otra parte, a nivel teórico y metodológico, estos estudios tienen el reto de complejizar su mirada para así considerar las múltiples relaciones existentes entre el género y la sexualidad y otros factores estructurantes como la etnicidad, la edad, las lógicas económicas o el origen socio-cultural. Del mismo modo que muchos de los trabajos etnográficos obvian los efectos de las relaciones de sexo-genéricas en sus objetos de análisis, los estudios sobre la sexualidad a menudo se limitan a analizar cuestiones comportamentales, relacionales e identitarias de sujetos y colectivos pertenecientes a minorías sexuales y de género, infravalorando o ignorando la relevancia de otros factores productores de desigualdades y violencias estructurales.

Las cuestiones económicas siempre tienen implicaciones socio-sexuales, esto es, si el sexo y el género son ante todo un hecho social, irremediablemente se ven afectados por las condiciones económicas y materiales de los sujetos y de las sociedades. Pensar la sexualidad y el género como elementos ajenos a las dinámicas económicas implica obviar los efectos de poder que se derivan de las relaciones capital-sexualidad y capital-género. De un modo similar, los estudios sobre diversidad sexo-genérica están obligados a superar las epistemologías de la blanquitud, que conciben y presentan a las personas LGTB como sujetos esencialmente blancos y pertenecientes al Norte global. En este sentido, los feminismos negros y decoloniales resultan fundamentales para inspirar perspectivas analíticas que incorporen las desigualdades raciales y étnicas, y visibilicen otras experiencias y expresiones que durante varias décadas han sido ignoradas tanto por la academia como por el activismo.

A pesar de estas dificultades y carencias, se ha de reconocer que en los últimos tiempos han proliferado las perspectivas que incorporan lo que se ha denominado la mirada interseccional, abordando cuestiones como la representación, la autoría y el lugar de enunciación de los trabajos etnográficos dentro de la especialidad. Aunque los debates sobre la representación y la autoría son ya clásicos en antropología, su incorporación a las investigaciones sobre diversidad sexual y de género ha estado marcada fundamentalmente por las interpelaciones de los feminismos negros y decoloniales. Para ello, ha sido importante la traducción al español de algunos de sus textos fundamentales, como la compilación Otras inapropiables revisada por Romero y García Dauder (Hooks et al. 2004), las traducciones del clásico Mujer, raza y clase de Angela Davis (2004) y de Cartografías de la diáspora de Avtar Brah (2011) o la antología Feminismos Negros, editada por Mercedes Jabardo (2012). 


\section{REFLEXIONES FINALES}

En España, el recorrido que han seguido los estudios antropológicos sobre la sexualidad y la diversidad sexual y de género ha estado marcado en los últimos treinta y siete años por tres factores clave: el contexto socio-político y académico, el influjo de las producciones internacionales y las sinergias establecidas con el feminismo.

En primer lugar, España ha experimentado un proceso de reconstitución democrática y aperturismo socio-sexual que la singulariza en cuanto a forma, tiempo y ritmos. Si bien es cierto que en buena parte de los países occidentales la represión social e institucional hacia las minorías sexo-genéricas no era mucho más laxa que en la España franquista, el régimen dictatorial español ejerció un control férreo no solo sobre las prácticas y expresiones públicas, sino también sobre los espacios de reflexión y producción del conocimiento crítico, la actividad política disidente y la vida privada. Es por ello que, tras la dictadura, se aceleraron los procesos sociales y políticos que permitieron la emergencia de discursos de liberación sexual. Para ello resultaron fundamentales las alianzas que activistas españoles establecieron en el extranjero, así como la influencia de discursos y luchas provenientes del resto de Europa y los Estados Unidos de América.

El contexto socio-político existente durante el régimen dictatorial franquista también influyó en el establecimiento de la antropología como disciplina académica. A pesar de que durante el franquismo existieron estudiosos e iniciativas que estimularon la mirada antropológica ${ }^{10}$, la institucionalización de la antropología española no se produjo hasta los últimos años del régimen. Y si retrocedemos más en el tiempo, este corto recorrido de la antropología española puede también explicarse por el hecho de que España, al no ser ya una potencia colonial, quedó fuera de la corriente europea decimonónica de la que surgió la antropología moderna. De este modo, mientras que en países como Francia o el Reino Unido se gestaban las bases científicas y teóricas de la disciplina, en España predominaba un folclorismo dedicado a recopilar datos relativos al patrimonio cultural nacional (Gazeta de Antropología 1984). Por tanto, la antropología en España cuenta con una historia reciente con respecto a otros países europeos y ha necesitado construir su propio recorrido de consolidación para significarse dentro del campo de las ciencias sociales.

En segundo lugar, es indiscutible la influencia de autores y autoras extranjeros, así como de los debates derivados de algunas corrientes de pensamiento internacionales. Este es el caso del post-estructuralismo francés, las teorías queer y los transgender studies norteamericanos, o los feminismos negros anglosajones, corrientes todas ellas surgidas en territorios de referencia para muchos campos de las ciencias sociales, pero también de otras, como los estudios decoloniales, que se han desarrollado en zonas a menudo infravaloradas por los centros de producción académica internacional. Sin lugar a dudas, la antropología de la sexualidad en España se ha desarrollado en buena medida gracias a estos influjos, lo que le ha permitido incorporarse a los debates y disputas académicas de ámbito global.

Finalmente, hay que destacar la influencia de la antropología feminista española en el desarrollo de los estudios sobre diversidad sexual y de género. Los estudios feministas han aportado perspectivas teóricas y metodológicas, y también han contri-

${ }^{10}$ Es el caso del profesor Julián San Valero, quien imparte en 1950 un curso de doctorado sobre antropología cultural en la Universidad de Valencia que perdurará hasta 1983 (Aguirre 2015). 
buido a la apertura de un espacio propio en el que enmarcar las preocupaciones, debates y aportaciones que legitiman y caracterizan a la antropología de la sexualidad. Los espacios académicos de reflexión feminista han sido los que han dado cobertura a las iniciativas relacionadas con la diversidad sexual y de género dentro de la antropología, a través de sus grupos de investigación y espacios de debate. Las jornadas y congresos feministas han hecho suyas las preocupaciones de la antropología que estudia la diversidad sexo-genérica, mientras que, a su vez, los antropólogas y antropólogos de esta especialidad se han autodefinido en muchas ocasiones dentro de los marcos conceptuales, metodológicos e ideológicos del feminismo. Estas relaciones han sido posibles gracias a la emergencia de nuevos paradigmas feministas que se alejan de la noción esencial, universal y monolítica de la categoría mujer, para interesarse por las interrelaciones entre diversas formas de opresión y las alianzas estratégicas, lo que ha permitido ampliar el objeto clásico del feminismo. En el caso español, las contribuciones de algunas feministas, como Cristina Garaizábal y Empar Pineda, han resultado esenciales para la articulación de la lucha contra la patologización de las personas trans. Al mismo tiempo, el compromiso político y análisis de algunas personas trans, como Miquel Missé, Pol Galofré o Amets Suess, ha sido importante para configurar un feminismo diverso e inclusivo, sensible a la multiplicidad de expresiones corporales y a la superposición de mecanismos de identificación y opresión. En este cruce de caminos entre feminismo y diversidad han emergido nuevas corrientes como los transfeminismos y las perspectivas queer o decoloniales.

Por todo lo dicho, resulta necesario construir la historia de los estudios sobre la sexualidad en antropología desde contextos particulares, precisamente para no asumir otras historias como propias sin tener en cuenta las singularidades locales. La historia de esta especialidad antropológica en Estados Unidos, Inglaterra o Francia no es la misma que la de España. Aunque existen influencias, sinergias y semejanzas, no necesariamente se reproducen los mismos recorridos, debates ni interrogaciones. La diversidad sexo-genérica es un universo analítico vasto y variopinto al que merece la pena prestar atención desde una perspectiva situada que atienda a las particularidades y permita comprender los fenómenos sociales en un determinado contexto. No cabe duda de que pueden identificarse itinerarios compartidos y espacios de discusión común que potencian el debate antropológico con otros colegas del resto del mundo y contribuyen al entendimiento de la realidad social. No obstante, no deberíamos abandonar una mirada local a la hora de entender las dinámicas globales. La antropología no está en condiciones de caer en los universalismos sexuales y de género que acostumbran a invisibilizar la diversidad humana, a naturalizar las perspectivas hegemónicas y a legitimar las dinámicas de poder que impregnan al sexo y al género.

\section{BIBLIOGRAFÍA CITADA}

Acebrón Ruiz, Julian y Rafael M. Mérida Jiménez (eds.). 2006. Diàlegs gais, lesbians, queer. Lleida: Edicions de la Universitat de Lleida.

Adam Donat, Antoni y Àlvar Martínez Vidal. 2008. "Infanticidas, violadores, homosexuales y pervertidos de todas las categorías. La homosexualidad en la psiquiatría del franquismo", en Javier Ugarte (ed.), Una discriminación universal. La bomosexualidad bajo el franquismo y la transición: 109-138. Barcelona/Madrid: Egales. 
Aguirre, Ángel. 2015. “Un siglo de antropología española”, en Nuria Fernández Moreno (comp.), Antropología y comparación cultural. Métodos y teorías: 51-67. Madrid: UNED.

Arnalte, Arturo. 2003. Redada de Violetas. La representación de los homosexuales durante el franquismo. Madrid: La esfera.

Azpiazu, Jokin. 2017. Masculinidades y Feminismo. Barcelona: Virus.

Barbé i Serra, Alba. 2013. "Ésser al llindar. La performance drag king: Un indret en pugna per establir nous i diferents ordres sociosexuals". Quaderns de l'Institut Català d'Antropologia 18(1): 1-18.

Barbé i Serra, Alba. 2015. El cross-dressing en el context català del segle XXI. Tesis doctoral. Barcelona: Departamento de Antropología Social, Universitat de Barcelona.

Benedict, Ruth. 1939. "Sex in Primitive Society". American Journal of Orthopsychiatry 9(3): 570-573.

Berná, David. 2016. Subjetividad y Resistencia desde los Márgenes: Procesos de articulación identitaria entre los gitanos y gitanas LGTB. Tesis doctoral. Madrid: Departamento de Antropología, Universidad Autónoma de Madrid.

Blanco López, Juan y José María Valcuende del Río (eds.). 2003. Hombres: la construcción cultural de las masculinidades. Madrid: Talasa.

Boellstorff, Tom. 2007. "Queer Studies in the House of Anthropology". Annual Review of Anthropology 36: $17-35$.

Bonal, Raimon, Teresa Climent y Joan Costa. 1985. Aproximació sociològica a l'homosexualitat: una anàlisi feta sobre la realitat catalana. Barcelona: Institut Lambda.

Brah, Avtar. 2011. Cartografías de la diáspora. Identidades en cuestión. Madrid: Traficantes de sueños.

Burgos Díaz, Elvira. 2013. "El escándalo de lo humano: lesbianas y mujeres", en Beatriz Suárez Briones (ed.), Las lesbianas (no) somos mujeres. En torno a Monique Wittig: 51-84. Barcelona: Icaria.

Burgos Díaz, Elvira. 2014. "La vida en las identidades", en Beatriz Suárez Briones (ed.), Feminismos lesbianos y queer. Representación, visibilidad y politicas: 71-82. Madrid: Plaza y Valdés.

Butler, Judith. 1990. Gender Trouble: Feminism and the Subversion of Identity. Nueva York/Londres: Routledge.

Butler, Judith. 1993. Bodies that Matter: On the Discursive Limits of "Sex". Nueva York/Londres: Routledge.

Cáceres Feria, Rafael. 2013. "Diversidad sexual: contextos locales, discursos globales", en José María Valcuende del Río, Maria Marco Macarro y David Alarcón Rubio (eds.), Diversidad sexual en Iberoamérica: 15-25. Sevilla: Aconcagua.

Cáceres Feria, Rafael y José María Valcuende del Río. 2014. “Globalización y diversidad sexual, gays y mariquitas en Andalucía". Gazeta de Antropologia 30(3): art. 7. Disponible en: http:// hdl.handle.net/10481/33814

Calle, Jaime de la. 2011. "Lecturas de "antropología de la sexualidad" como acontecimiento intelectual". Revista de Investigaciones en Educación 9(2): 99-115.

Calvo Borobia, Kerman. 2003. "Actitudes sociales y homosexualidad en España", en Oscar Guasch Andreu y Olga Viñuales (eds.), Sexualidades: diversidad y control social: 299-320. Barcelona: Bellaterra.

Calvo Borobia, Kerman. 2010. "Reconocimiento, ciudadanía y políticas públicas hacia las uniones homosexuales en Europa”. Revista Española de Investigaciones Sociológicas 129: 37-59.

Capel, Horacio. 2009. "La antropología española y el magisterio de Claudio Esteva Fabregat. Estrategias institucionales y desarrollo intelectual en las disciplinas científicas". Scripta Nova 13(287): $1-98$.

Cardín, Alberto. 1984. Guerreros, chamanes y travestis: indicios de homosexualidad entre los exóticos. Barcelona: Tusquets.

Cardín, Alberto. 1991. SIDA: enfoques alternativos. Barcelona: Laertes.

Cardín, Alberto y Armand de Fluvià. 1985. SIDA. ¿Maldición bíblica o enfermedad letal? Barcelona: Laertes.

Carlavilla, Mauricio. 1956. Sodomitas. Homosexuales, políticos, científicos, criminales, espias, etc. Madrid: Nos.

Cleminson, Richard y Francisco Vázquez García. 2007. "Los invisibles". A History of Male Homosexuality in Spain, 1850-1940. Cardiff: University of Wales Press. 
Coll-Planas, Gerard. 2010. La Voluntad y el deseo. La construcción social del género y la sexualidad: el caso de lesbianas, gays y trans. Barcelona/Madrid: Egales.

Coll-Planas, Gerard. 2012. La Carne y la metáfora: una reflexión sobre el cuerpo en la teoría queer. Barcelona/Madrid: Egales.

Comas d'Argemir, Dolors. 1990. Vides de dona: treball, família i sociabilitat entre les dones de classes populars: 1900-1960. Barcelona: Fundació Serveis de Cultura Popular.

Comas d'Argemir, Dolors. 1995. Trabajo, género, cultura: la construcción de desigualdades entre hombres y mujeres. Barcelona: Institut Català d'Antropologia.

Córdoba García, David, Javier Sáez y Paco Vidarte (eds.). 2005. Teoría queer: políticas bolleras, maricas, trans, mestizas. Barcelona/Madrid: Egales.

Davis, Angela. 2004. Mujer, raza y clase. Madrid: Akal.

De Lauretis, Teresa. 1994. Technologies of Gender: Essays on Theory, Film, and Fiction. Houndmills: Macmillan.

Delgado, Manuel. 1992. "Alberto Cardín, escritor, antropólogo y destacado agitador cultural, muere en Barcelona a los 44 años". El País. Disponible en: <https://elpais.com/diario/1992/01/27/cultura/696466801_850215.html>. Fecha de acceso: 31 jul. 2017.

Donoso López, Silvia. 2006. "Maternidad lésbica: reconocimiento y soporte en el ámbito de las relaciones familiares", en Xavier Roigé (ed.), Familias de ayer, familias de hoy: continuidades y cambios en Cataluña: 515-534. Barcelona: Icaria.

Donoso López, Sivia. 2012. La familia lesboparental, ¿reinvención de la familia?. Tesis doctoral. Barcelona: Departamento de Antropología Social, Universitat de Barcelona.

Enguix Grau, Begonya. 1995. Poder y deseo: la homosexualidad masculina en Valencia. Valencia: Edicions Alfons el Magnànim.

Enguix Grau, Begonya. 2010. "Fronteras, cuerpos e identidades gays". Quaderns-e de l'ICA 26: 83-106.

Enguix, Grau, Begonya. 2012. "Cultivando cuerpos, modelando masculinidades". Revista de Dialectologia y Tradiciones Populares 67(1): 147-180.

Enguix Grau, Begonya. 2017. "Protesta, mercado e identidad en las celebraciones del Orgullo LGTB en España". Convergencia: Revista de ciencias sociales 73 (enero-abril): 165-186.

Esteban Galarza, Mari Luz. 2004. Antropología del cuerpo: género, itinerarios corporales, identidad y cambio. Barcelona: Bellaterra.

Esteban Galarza, Mari Luz. 2011. Crítica del pensamiento amoroso: temas contemporáneos. Barcelona: Bellaterra.

Falguera Ríos, Mercè. 2013. "Una madre, dos madres: Las maternidades de las mujeres lesbianas", en José María Valcuende del Río, María J. Marco Macarro y David Alarcón Rubio (coords.), Estudios sobre diversidad sexual en Iberoamérica: 355-362. Sevilla: Aconcagua.

Fausto-Sterling, Anne. 2000. Sexing the body: gender politics and the construction of sexuality. Nueva York: Basic Books.

Foucault, Michel. 1976. Histoire de la sexualité I. La volonté de savoir. París: Gallimard.

Frankis, Jamie y Paul Flowers. 2009. “Public Sexual Cultures: A Systematic Review of Qualitative Research Investigating Men's Sexual Behaviors with Men in Public Spaces". Journal of Homosexuality 56(7): 861-893. doi: 10.1080/00918360903187846

Galofré, Pol y Miquel Missé (eds.). 2015. Políticas trans: Una antología de textos desde los estudios trans norteamericanos. Barcelona/Madrid: Egales.

Gazeta de Antropología. 1984. "La antropología en España”. Gazeta de Antropología 3: Editorial. Disponible en: <http://hdl.handle.net/10481/13791>.

Goytisolo, Juan. 1985. Coto vedado. Barcelona: Seix Barral.

Gregori Flor, Núria. 2006. "Los cuerpos ficticios de la biomedicina. El proceso de construcción del género en los protocolos médicos de asignación de sexo en bebés intersexuales". AIBR. Revista de Antropología Iberoamericana 1(1): 103-124.

Gregori Flor, Núria. 2013. “Utopías dicotómicas sobre los cuerpos sexuados”. Arbor. Ciencia, Pensamiento y Cultura 189(763): a071. doi: 10.3989/arbor.2013.763n5008

Gregorio Gil, Carmen. 1998. Migración femenina: su impacto en las relaciones de género. Madrid: Narcea. 
Gregorio Gil, Carmen. 2014. "Traspasando las fronteras dentro-fuera: Reflexiones desde una etnografía feminista". AIBR Revista de Antropologia Iberoamericana 9(3): 297-322. doi: 10.11156/ aibr.090305

Guasch Andreu, Óscar. 1993. "Para una sociología de la sexualidad”. Reis: Revista española de investigaciones sociológicas 64: 105-122.

Guasch Andreu, Óscar. 2000. La Crisis de la heterosexualidad. Barcelona: Laertes.

Guasch Andreu, Óscar. 2006. Héroes, científicos, heterosexuales y gays: Los varones en perspectiva de género. Barcelona: Edicions Bellaterra.

Guasch Andreu, Óscar y Eduardo Lizardo. 2017. Chaperos: precariado y prostitución homosexual. Barcelona: Bellaterra.

Guasch Andreu, Óscar y Jordi Mas Grau. 2014. "Bodily, Gender, and Identity Projects in Spain: From the Transvestite to the Transsexual, , en Rafael Mérida Jimenez (ed.), Hispanic (LGT) Masculinities in Transition: 51-64. Nueva York: Peter Lang.

Guasch Andreu, Óscar y Olga Viñuales (eds.). 2003. Sexualidades: diversidad y control social. Barcelona: Bellaterra.

Haraway, Donna. 1991. Simians, cyborgs, and women: the reinvention of nature. Nueva York/Londres: Routledge.

Herdt, Gilbert (ed.). 1992. Homosexualidad ritual en Melanesia. José C. Lisón Arcal (trad.). Madrid: Fundación Universidad-Empresa.

Herdt, Gilbert. 1997. Same Sex, Different Cultures: Exploring Gay and Lesbian Lives. Boulder: Westview Press.

Hooks, Bell, Avtar Brah, Chela Sandoval, Gloria Anzaldúa, Aurora Levins Morales, Kum-Kum Bhavnani, Margaret Coulson, M. Jacqui Alexander y Chandra Talpade Mohanty. 2004. Otras inapropiables. Feminismos desde las fronteras. Carmen Romero Bachiller y Silvia García Dauder (rev.). Madrid: Traficantes de sueños.

Huard, Geoffroy 2014. Los antisociales: historia de la homosexualidad en Barcelona y París, 19451975. Madrid: Marcial Pons Historia.

Iglesias de Ussel, Julio. 1983. "La Sociología de la sexualidad en España". REIS Revista Española de Investigaciones Sociológicas 21: 103-133.

Imaz, Elixabete. 2010. Convertirse en madre: etnografía del tiempo de gestación. Madrid: Cátedra. Jabardo, Mercedes (ed.). 2012. Feminismos negros. Una antología. Madrid: Traficantes de sueños.

Juliano, Dolores. 1992. El juego de las astucias: mujer y construcción de modelos sociales alternativos. Madrid: Horas y horas.

Juliano, Dolores. 1998. Las que saben: subculturas de mujeres. Madrid: Horas y horas.

Llamas, Ricardo. 1995. Construyendo sidentidades: estudios desde el corazón de una pandemia. Madrid: Siglo XXI.

Llamas, Ricardo. 1998. Teoría torcida: prejuicios y discursos en torno a "la homosexualidad". Madrid: Siglo XXI.

Langarita Adiego, Jose Antonio. 2015. En tu árbol o en el mío: Una aproximación etnográfica a la práctica del sexo anónimo entre hombres. Barcelona: Bellaterra.

Laqueur, Thomas. W. 1990. Making Sex: Body and Gender from the Greeks to Freud. Cambridge: Harvard University Press.

López Ibor, Juan José. 1968. El libro de la vida sexual. Barcelona: Danae.

Lores Masip, Fernando. 2012. Deseo y Peligro. Anotaciones antropológicas a una teoría de la contaminación y de los cuidados sexuales. Tesis doctoral. Madrid: Departamento de Antropología Social, Universidad Complutense de Madrid.

Lores Masip, Fernando y Ariadna Ayala Rubio. 2015. "Entre el "deseo" de ser padres y ser padres "de tercera": experiencias de la monoparentalidad entre los padres solteros por elección (PSPE)", en María Isabel Jociles Rubio y Raquel Medina Plana (coords.), La monoparentalidad por elección: el proceso de construcción de un modelo de familia: 143-184. Valencia: Tirant Lo Blanch.

Malinowski, Bronislaw. 1927. Sex and Repression in Savage Society. Londres: Routledge and Kegan Paul.

Malinowski, Bronislaw. 1929. The sexual life of Savages in North-Western Melanesia: An Ethnographic Account of Courtship, Marriage and Family Life among the Natives of the Trobriand island, British New Guinea. Nueva York/Londres: Routledge. 
Malinowsky, Bronislaw. 1989. Diario de campo en Melanesia. Barcelona: Júcar.

Martín Gaite, Carmen. 1992. Nubosidad variable. Barcelona: Anagrama.

Mas Grau, Jordi. 2014. Subjetividades y cuerpos gestionados. Un estudio sobre la patologización y medicalización del transgénero. Tesis doctoral. Barcelona: Departamento de Antropología Social, Universitat de Barcelona.

Mas Grau, Jordi. 2015. "Transexualidad y transgenerismo. Una aproximación teórica y etnográfica a dos paradigmas enfrentados". Revista de Dialectología y Tradiciones Populares 70(2): 485-501. doi: $10.3989 /$ rdtp.2015.02.009

Mas Grau, Jordi. 2017. "Del transexualismo a la disforia de género en el DSM. Cambios terminológicos, misma esencia patologizante”. Revista Internacional de Sociología 75(2): e059. doi: 10.3989/ ris.2017.75.2.15.63

Mead, Margaret. 1950. Sex and Temperament in Three Primitive Societies. Nueva York: The New American Library.

Mejía, Norma. 2006. Transgenerismos: una experiencia transexual desde la perspectiva antropológica. Barcelona: Bellaterra.

Mendicutti, Eduardo. 1982. Una mala noche la tiene cualquiera. Barcelona: Tusquets.

Mérida Jiménez, Rafael M. (ed.). 2002. Sexualidades transgresoras: una antología de estudios queer. Barcelona: Icaria.

Mérida Jiménez, Rafael M. 2008. Damas, santas y pecadoras: hijas medievales de Eva. Barcelona: Icaria.

Mérida Jiménez, Rafael M. 2009a. Cuerpos desordenados. Barcelona: UOC.

Mérida Jiménez, Rafael M. (ed.). 2009b. Manifiestos gays, lesbianos y queer: testimonios de una lucha (1969-1994). Barcelona: Icaria.

Mérida Jiménez, Rafael M. (ed.). 2013. Minorías sexuales en España (1970-1995): textos y representaciones. Barcelona: Icaria.

Mérida Jiménez, Rafael M. 2016. Transbarcelonas: cultura, género y sexualidad en la España del siglo XX. Barcelona: Bellaterra.

Mérida Jiménez, Rafael. M. y Jose Luís Peralta (eds.). 2015. Las masculinidades en la Transición. Barcelona/Madrid: Egales.

Mira, Alberto. 2004. De Sodoma a Chueca: una bistoria cultural de la homosexualidad en España en el siglo $X X$. Barcelona/Madrid: Egales.

Negre, Pere. 1988. La prostitución popular: relatos de vida. Barcelona: Fundació Caixa de Pensions.

Nieto Piñeroba, José Antonio. 1989. Cultura y sociedad en las prácticas sexuales. Madrid: Fundación Universidad-Empresa.

Nieto Piñeroba, José Antonio (ed.). 1991. La sexualidad en la sociedad contemporánea: lecturas antropológicas. Madrid: Fundación Universidad-Empresa.

Nieto Piñeroba, José Antonio. 1993. Sexualidad y deseo: crítica antropológica de la cultura. Madrid: Siglo XXI.

Nieto Piñeroba, José Antonio (ed.). 1998. Transexualidad, transgenerismo y cultura: antropología, identidad y género. Madrid: Talasa.

Nieto Piñeroba, José Antonio (ed.). 2003. Antropología de la sexualidad y diversidad cultural. Madrid: Talasa.

Nieto Piñeroba, José Antonio. 2008. Transexualidad, intersexualidad y dualidad de género. Barcelona: Bellaterra.

Nieto Piñeroba, José Antonio. 2011. Sociodiversidad y sexualidad. Madrid: Talasa.

Osborne, Raquel. 1991. Las prostitutas: una voz propia: crónica de un encuentro. Barcelona: Icaria.

Pichardo Galán, José Ignacio. 2009. Entender la diversidad familiar: relaciones bomosexuales y nuevos modelos de familia. Barcelona: Bellaterra.

Pichardo Galán, José Ignacio, Matías de Stéfano Barbero y M. Laura Martín-Chiappe. 2015. "(Des)naturalización y elección: emergencias en la parentalidad y el parentesco de lesbianas, gays, bisexuales y transexuales". Revista de Dialectología y Tradiciones Populares 70(1): 187-203. doi: 10.3989/rdtp.2015.01.009

Platero, R. Lucas. 2008. Lesbianas: discursos y representaciones. Barcelona: Melusina.

Platero, R. Lucas (ed.). 2012. Intersecciones: cuerpos y sexualidades en la encrucijada. Barcelona: Bellaterra. 
Plummer, Ken. 1984. "Sexual Diversity: A Sociological Perspective", en Kevin Howells (ed.), The Psychology of Sexual Diversity: 219-253. Oxford: Blackwell Publisher.

Prat i Carós, Joan. 1992. Antropología y etnología. Las ciencias sociales en España. Historia inmediata, crítica y perspectivas. Madrid: Caja de Ahorros y Monte de Piedad de Madrid.

Preciado, Paul B. 2002. Manifiesto contrasexual. Barcelona: Opera Prima.

Preciado, Paul B. 2008. Testo yonqui. Madrid: Espasa.

Rodó de Zárate, Maria. 2013. "Gènere, cos i sexualitat. La joventut, l'experiència i l'ús de l'espai públic urbàn. Papers: Revista de Sociologia 98(1): 127-142.

Rodó de Zárate, Maria. 2015. "Young Lesbians Negotiating Public Space: An Intersectional Approach through Places". Children's Geographies 13(4): 413-434. doi:10.1080/14733285.2013.848741

Rodríguez, Josep A. 1985. "Homosexualidad: Una enfermedad sin nombre”. Sistema. Revista de Ciencias Sociales 64: 83-103.

Roigé, Xavier (ed.). 2006. Familias de ayer, familias de boy: continuidades y cambios en Cataluña. Barcelona: Icaria.

Romero Bachiller, Carmen, Silvia García Dauder y Carlos Bargueiras Martínez (eds.). 2005. El eje del mal es heterosexual: figuraciones, movimientos y prácticas feministas queer. Madrid: Traficantes de sueños.

Rubin, Gayle. 1975. "The Traffic in Women: Notes on the "Political Economy" of Sex", en Rayna Reiter (ed.), Toward an Anthropology of Women: 157-210. Nueva York: Monthly Review Press.

Rubin, Gayle. 1984. "Thinking Sex. Notes for Radical Theory of Politics of Sexuality", en Carole S. Vance (ed.), Pleasure and Danger. Exploring Female Sexuality: 267-319. Nueva York/Londres: Routledge.

Rubin, Gayle 1986. "Tráfico de mujeres: notas sobre la "economía política" del sexo". Revista Nueva Antropología VIII(30): 95-145.

Rubin, Gayle. 1989. "Reflexionando sobre el sexo: notas para una teoría radical de la sexualidad", en Carole S. Vance (ed.), Placer y peligro: explorando la sexualidad femenina (trads.Julio Velasco y M. ${ }^{a}$ Ángeles Toda): 113-190. Madrid: Talasa.

Sabater Tomas, Antonio. 1962. Gamberros, homosexuales, vagos y maleantes: estudio jurídico-sociológico. Barcelona: Hispano Europea.

Sáez, Javier y Sejo Carrascosa. 2011. Por el culo. Políticas anales. Barcelona/Madrid: Egales.

Sáez Jiménez, Fernando y Olga Viñuales. 2007. Armarios de cuero: relatos de vida. BDSM. Barcelona: Bellaterra.

Sedgwick, Eve Kosofsky. 1990. Epistemology of the Closet. Berkeley: University of California Press.

Solá, Miriam y Elena Urko (eds.). 2013. Transfeminismos: epistemes, fricciones y flujos. Tafalla: Txalaparta.

Soley-Beltran, Patricia. 2009. Transexualidad y la matriz heterosexual: un estudio crítico de Judith Butler. Barcelona: Bellaterra.

Stolcke, Verena. 1992. Racismo y sexualidad en la Cuba colonial. Madrid: Alianza.

Stolcke, Verena. 2000. «Es el sexo para el género lo que la raza para la etnicidad... y la naturaleza para la sociedad?". Política y Cultura 14: 25-60.

Stryker, Susan y Aren Z. Aizura (eds.). 2013. The Transgender Studies Reader 2. Nueva York/Londres: Routledge.

Stryker, Susan y Stephen Whittle (eds.). 2006. The Transgender Studies Reader. Nueva York/Londres: Routledge.

Suárez Briones, Beatriz (ed.). 2013. Las Lesbianas (no) somos mujeres: en torno a Monique Wittig. Barcelona: Icaria.

Suárez Briones, Beatriz (ed.). 2014. Feminismos lesbianos y queer: representación, visibilidad y politicas. Madrid: Plaza y Valdés.

Tiefer, Leonore. 1995. Sex is not a Natural Act and other Essays. Nueva York: Westview Press.

Torras, Meri (ed.). 2007. Cuerpo e identidad: estudios de género y sexualidad I. Bellaterra: Institut Català de les Dones.

Torras, Meri (ed.). 2011. Accions i reinvencions: cultures lèsbiques a la Catalunya del tombant de segle XX-XXI. Barcelona: Editorial UOC.

Trujillo Barbadillo, Gracia. 2009. Deseo y resistencia: treinta años de movilización lesbiana en el Estado español (1977-2007). Barcelona/Madrid: Egales. 
Tusquets, Esther. 1978. El mismo mar todos los veranos. Barcelona: Lumen.

Ugarte Pérez, Javier (coord.). 2008. Una Discriminación universal: la homosexualidad bajo el franquismo y la Transición. Barcelona/Madrid: Egales.

Ugarte Pérez, Javier. 2014. Placer que nunca muere: sobre la regulación del homoerotismo occidental. Barcelona/Madrid: Egales.

Valcuende del Río, José María. 2006. "De la heterosexualidad a la ciudadania". AIBR. Revista de Antropología Iberoamericana 1(1): 125-142. doi: 10.11156/aibr.010109

Valcuende del Río, José María y Assumpta Sabuco i Cantó. 2003. "La "homosexualidad" como representación hiperbólica de la masculinidad", en Juan Blanco y José María Valcuende del Río (eds.), Hombres: la construcción cultural de las masculinidades: 135-154. Madrid: Talasa.

Valle, Teresa del (ed.). 1993. Gendered Anthropology. Nueva York/Londres: Routledge.

Valle, Teresa del (ed.). 2000. Perspectivas feministas desde la antropología social. Barcelona: Ariel.

Valle, Teresa del y Carmela Sanz Rueda. 1991. Género y sexualidad. Madrid: Fundación Universidad-Empresa.

Vallejo Nágera, Antonio. 1937. Eugenesia de la hispanidad y regeneración de la raza. Burgos: Editorial Española.

Vallejo Nágera, Antonio. 1941. Tratamiento de las enfermedades mentales. Valladolid: Librería Santarén.

Vartabedian Cabral, Julieta. 2012. Geografía travesti: Cuerpos, sexualidad y migraciones de travestis brasileñas (Rio de Janeiro-Barcelona). Tesis doctoral. Barcelona: Departamento de Antropología Social, Universitat de Barcelona.

Vartabedian Cabral, Julieta. 2013. "Tengo mucho placer para enseñarte: Sobre travesties brasileñas trabajadoras del sexo y la gestión pública de la prostitución en Barcelona". Quaderns-e de l'Institut Catala d'Antropologia 18(1): 80-97.

Vartabedian Cabral, Julieta. 2014. “Sobre travestis, clientes y maridos: Género y sexualidad en la construcción de las identidades de travestis Brasileñas trabajadoras del sexo". Revista de Antropología Social 23: 237-261. doi: 10.5209/rev_raso.2014.v23.46734

Vázquez García, Francisco y Andrés Moreno Mengíbar. 1997. Sexo y razón: una genealogía de la moral sexual en España, siglos XVI-XX. Madrid: Akal.

Vázquez Montalbán, Manuel. 1987. Los alegres muchachos de Atzavara. Barcelona: Seix Barral.

Vendrell i Ferré, Joan. 1994. "Estratègies i tècniques etnogràfiques en antropologia sexual: principis generals i problemàtica específican. Revista d'Etnologia de Catalunya 5: 134-147.

Vendrell i Ferré, Joan. 1999. Pasiones ocultas: de cómo nos convertimos en sujetos sexuales. Barcelona: Ariel.

Vendrell i Ferré, Joan. 2009. “Corregir el cuerpo o cambiar el sistema? La transexualidad ante el orden de género". Sociológica 24(69): 61-78.

Vidarte, Paco. 2007. Ética marica: proclamas libertarias para una militancia LGTBQ. Barcelona/ Madrid: Egales.

Villaamil Pérez, Fernando. 2004. La transformacion de la identidad gay en España. Madrid: Los Libros de la Catarata.

Villaamil Pérez, Fernando. 2013. "Ciudadanías sexuales frágiles: Información, carne y VIH". Quaderns de l'Institut Catala d'Antropologia 18(1): 19-38.

Villaamil Pérez, Fernando. 2016. "Ponte una medalla. Experiencias gais y ciudadanía frente al VIH", en Rafael Mérida Jiménez (ed.), Masculinidades disidentes: 57-88. Barcelona: Icaria.

Villaamil Pérez, Fernando y M. Isabel Jociles Rubio. 2008. "Diferencias y desigualdades entre los HSH usuarios de locales comerciales de encuentro sexual: algunas contribuciones a las estrategias comunitarias de prevención del VIH". Etnográfica 12(2): 285-521. doi: 10.1177/ 1363460711415334

Villaamil Pérez, Fernando y M. Isabel Jociles Rubio. 2011. "Risk and community: The impact of HIV among gays in Madrid. The case of sex clubs". Sexualities 14(5): 580-596. doi: 10.1177/ 1363460711415334

Villaamil Pérez, Fernando, M. Isabel Jociles Rubio y Fernando Lores Masip. 2005. “La concepción del sujeto en los estudios sobre el riesgo y la prueba del VIH en $\mathrm{HSH}$. Revista Internacional de Sociología 42: 69-99. doi: 10.3989/ris.2005.i42.197 
Villena, Luis Antonio de. 1982. Ante el espejo. Barcelona: Argos Vergara.

Villena, Luis Antonio de. 1989. Chicos. Madrid: Mondadori.

Viñuales, Olga. 2000. Identidades lésbicas: discursos y prácticas. Barcelona: Bellaterra.

Weeks, Jeffrey. 1985. Sexuality and its Discontents: Meanings, Myths, and Modern Sexualities. Nueva York/Londres: Routledge.

Weston, Kath. 1993. "Lesbian / Gay Studies in the House of Anthropology". Annual Review of Anthropology 22: 339-367. doi: 10.1146/annurev.an.22.100193.002011

Weston, Kath. 2003. Las familias que elegimos. Lesbianas, gays y parentesco. Barcelona: Bellaterra.

Fecha de recepción: 3 de julio de 2017

Fecha de aceptación: 2 de octubre de 2017 